Voix et Images

voixetimages

\title{
Voix et Images, mais de quel pays?
}

\section{Pierre Hébert}

Volume 13, numéro 3 (39), printemps 1988

Jack Kerouac et l’imaginaire québécois

URI : https://id.erudit.org/iderudit/200739ar

DOI : https://doi.org/10.7202/200739ar

Aller au sommaire du numéro

\section{Éditeur(s)}

Université du Québec à Montréal

\section{ISSN}

0318-9201 (imprimé)

1705-933X (numérique)

Découvrir la revue

\section{Citer cet article}

Hébert, P. (1988). Voix et Images, mais de quel pays? Voix et Images, 13(3), 492-496. https://doi.org/10.7202/200739ar d'utilisation que vous pouvez consulter en ligne.

https://apropos.erudit.org/fr/usagers/politique-dutilisation/ 


\section{Roman}

\section{Voix et images, mais de quel pays?}

\section{par Pierre Hébert, Université de Toronto}

Au pays de Québec, rien ne doit mourir, certes, mais beaucoup de choses ont changé. Ne faut-il pas s'étonner, par exemple, d'entendre un certain politicien affirmer, récemment, qu'il n'y a pas de honte à être nationaliste? C'est comme lorsque Camus disait qu'il n'y a pas de honte à être heureux: comment peut-il en effet y avoir de la gêne à vivre des valeurs fondamentales?

À cet égard, le rapport du roman au pays a lui aussi beaucoup changé: mais bien malin celui ou celle qui pourrait déceler une voix, une image claires! Un seul groupe politique, un seul mouvement idéologique ou littéraire ne peut 
rassembler tous les élans; on ne s'abrite plus sous un même parapluie. Prenons pour témoin ces trois romans volumineux de Louis-Martin Tard, Ronald Lavallée et Victor-Lévy Beaulieu: il est difficile d'imaginer des auvres aussi différentes dans leur lieu commun avec le Québec.

\section{Un titre séduisant}

Aussi bien le dire tout de suite: je cède devant l'énorme tâche de résumer ces trois cuvres, de 500 pages chacune. Pour ce roman de Louis-Martin Tard, Il y aura toujours des printemps en Amérique ${ }^{1}$, qu'il suffise de dire que l'objectif premier du romancier est ambitieux: suivre, de 1633 à nos jours, les combats, les rêves d'une famille et de ses descendants, les Malouin. Louis-Martin Tard repasse à travers toute l'histoire du Québec, en mineur et en majeur: de la Conquête aux événements d'octobre 1970, de l'arrivée de La Capricieuse au débarquement de Dieppe. Un fil rattache néanmoins tous ces épisodes: nous devons rester ici, où nous avons bâti maison, quoi qu'il arrive (p. 45).

Il y a de ces titres qui parlent: en voici un. Car on comprend tout de suite que le roman ne va pas que nous raconter une histoire, avec un grand ou un petit $\mathrm{H}$; qu'il ne va pas non plus nous imposer une longue complainte passéiste. Tout au contraire, Il y aura toujours des printemps en Amérique vise à nous transmettre, à travers mais aussi au-delà même du temps, un sentiment de continuité et de nécessité historique. Cette transmission d'un savoir débouche sur une manière d'être; elle n'est d'ailleurs pas sans rappeler le canard de bois de Louis Caron, transmis chez les Bellerose: un canard, c'est plus qu'un canard, c'est en l'occurrence la famille Malouin et ses 350 ans en Amérique. L'unité généalogique l'emporte même sur l'unité narrative. En fait, la narrativité est fort ténue: le roman a tellement de choses à montrer qu'il prend parfois à peine le temps de les raconter. La gageure est donc de retenir l'intérêt du lecteur par l'envergure du parcours historique, par la sympathie envers les personnages, plutôt que par l'intérêt narratif proprement dit; même les «grands faits» de l'histoire sont la plupart du temps ramenés à la perception qu'en ont les personnages. Ce projet ambitieux doit cependant nous retenir dans son essence même: celui d'un passé assumé, compris, mais qui, en fin de récit, est projeté vers un avenir inconnu et illimité.

\section{On ne revient jamais chez soi}

Qui est Ronald Lavallée? Je l'ignore, n'ayant rien lu auparavant de cet auteur. Au surplus, j'ai devant moi cet énorme roman publié en France, écrit serré et coiffé d'un titre qui satisfait le compromis entre l'exotisme et le familier: Tchipayuk ou le chemin du loup 2 . Et la présentation de l'éditeur ne manque pas d'exploiter la veine: ce roman nous fait découvrir à travers son inoubliable héros un Canada inconnu où l'homme était encore partie intégrante de la nature. Dois-je me préparer à lire une fusion d'Agaguk et de Maria Chapdelaine? En vérité, il n'en est rien. Ronald Lavallée nous donne à lire un roman fascinant, dégagé d'un destinataire trop marqué et qui nous épargne de 
l'affligeant lexique final, même si ses personnages n'hésitent pas à utiliser des vrâ, icitte, couvarte.

Nous ne sommes pas dans le Grand Nord, mais bien dans l'Ouest canadien, chez les Métis, vers 1870-1885: voilà une avenue peu fréquentée. Le personnage principal, Askik Mercredi, est au début un jeune garçon de sept ans, dont la vie se déroulera en trois épisodes: l'enfance, la vie au Québec et le retour au pays natal. L'enfance, au Manitoba surtout, nous entraîne dans un monde peu familier. la vie d'un jeune Indien à travers la chasse au bison, le parcours des plaines et des forêts, la magie des aînés. Mais le jeune Askik caresse un rêve: s'instruire. C'est pourquoi il va au Québec, où l'attendent succès et revers mais, surtout, la seule vraie question: où se trouve la voie du bonheur? Askik décide alors de rentrer chez lui, mais le pays qu'il retrouve, il ne s'y reconnaît plus.

L'intérêt, la force du roman de Ronald Lavallée repose entre autres dans la variété des lectures qu'il invite. Ainsi, l'épisode de l'enfance d'Askik est narré au moyen d'un style descriptif minutieux, où le surnaturel garde une place essentielle. Point de hiatus entre les visions que l'on a, les histoires que l'on se raconte et la réalité vécue. Nous sommes ici en plein mythe, c'est-à-dire non pas une fabulation, mais plutôt une manière d'être, une formule de participation, de co-insertion entre l'homme et le monde où la continuité du visible et de l'invisible assure une harmonie véritablement universelle. Le divorce entre l'homme et le monde n'a pas encore eu lieu.

C'est au Québec que se fait la sortie du mythe. L'appât de la connaissance apportera à Askik un certain succès. Mais si une voie s'est dessinée pour Askik (devenu Alexis), c'est aux dépens d'une perte encore plus lourde, d'une désolidarisation. Car le prix à payer pour la connaissance, c'est aussi la solitude.

Mais le retour au pays natal, à la fin, sera aussi un échec: le mythe ne peut être réintégré, les vrais paradis étant ceux qu'on ne quitte jamais. Pourquoi alors Askik se dirige-t-il vers l'Alberta? Sans doute pour essayer d'y poursuivre, pour lui-même, cette vision d'enfance, le chemin du loup, qui mène aux plaines éternelles.

Tchipayuk nous présente, avec une écriture à l'occasion fort belle mais dans tous les cas sûre d'elle-même, un royaume inconnu, fascinant, l'Ouest, et un Québec qui prend une figure d'exil. Si un roman réussi permet à chacun d'y trouver son profit selon des attentes diverses, alors Tchipayuk me semble mériter ce qualificatif.

\section{Un homme et son péché}

Lorsque j'ai commencé ma lecture de l'Héritage ${ }^{3}$, de Victor-Lévy Beaulieu, je me suis demandé comment j'arriverais à terminer le premier tome, tellement le début me semblait laborieux; à mi-chemin dans le roman, j'avais hâte que paraisse le deuxième tome. Que s'était-il donc passé? 
Il faut dire en effet que le récit démarre plutôt lentement; sans doute convient-il de faire ses premiers pas avec prudence quand on veut mettre en branle une machine qui comptera, semble-t-il, 2000 pages. Mais, une fois le récit bien amorcé, il devient impossible d'y échapper. Et pourtant, les fidèles du téléroman le savent, point d'événements d'éclat dans cette histoire; s'il y eut un épisode déterminant, il s'est passé bien avant le début du récit, quatorze ans plus tôt, lorsque Xavier Galarneau a fait l'amour (?) à sa fille Miriam. C'est alors que le monde a chaviré, que Miriam s'est enfuie à Montréal, et que Xavier a entrepris de se punir, de se durcir tout au moins en se revêtant d'un discours rigoriste tout composé de sentences, de proverbes péremptoires. Ce n'est pas, non plus, l'intrigue de la petite histoire d'amour et de poésie entre Albertine et Philippe qui va nous garder le roman entre les mains. Mais alors, que se passe-t-il ici?

D'abord, les contrastes: Montréal et Trois-Pistoles, Loi et poème, agressivité et tendresse, autant d'images catégoriques qui donnent au roman du corps, des grosseurs. Mais, en même temps que ces images fortes, des finesses, des tendresses de sentiments, des rêves écrasés mais aussi vécus apparaissent de ça de là: tout ce mélange, à partir du milieu du roman, réussit à tisser un monde fascinant.

C'est d'ailleurs à partir de ce moment qu'une passion s'empare du roman. Mais c'est peut-être lorsqu'elle nous parle des mots que l'écriture atteint une intensité qu'en cette matière les lettres québécoises n'ont pas souvent fréquentée. Mais qu'on ne s'y méprenne pas! Victor-Lévy Beaulieu vibre intensément à l'écriture du plus vrai des poètes qu'est Philippe Couture: ce ne sont pas des mots tristes qui ne parlent que d'eux-mêmes, mais bien des mots qui habitent un espace, des désirs, une vie. Les mots fêtés ici sont ceux des écrivains du pays québécois, mais aussi d'ailleurs.

Mais quel est-il, ce pays de Victor-Lévy Beaulieu? Il ne s'agit pas, certes, d'un pays de consolation, c'est-à-dire d'une projection dans l'imaginaire sur fond de réel dégradé. Ce pays littéraire est ici bien réel: l'auteur ne pense pas que nommer les lieux suffise à se les approprier. L'entreprise se joue au niveau du littéraire, mais en exploitant l'entité des ressources disponibles: le roman crée, fait vivre des espaces inséparables des personnages et de leurs rêves. Ainsi Philippe et Albertine vivent-ils, dans leur moment d'extrême bonheur, à fleur d'un pays réel (p. 268) mais aussi fugitif, où l'enfance, la mer, Proust et Gaston Miron sont complices d'une vaste imagerie unifiée.

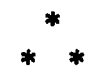

Les romans de L.-M. Tard, de R. Lavallée et de V.-L. Beaulieu, s'ils ont en commun un rapport essentiel à l'espace québécois, abordent celui-ci de manière très différente en ce qui a trait à leurs personnages. Chez L.-M. Tard, on sent que les personnages sont les acteurs d'un scénario tracé d'avance, l'histoire. Leur vie est précédée par une pièce qui s'est écrite pendant trois cent cinquante ans, et l'existence individuelle n'est pas véritablement une unité de compte. En revanche, 
chez R. Lavallé, face au groupe social qui exerce une pression forte, que ce groupe soit métis ou québécois, Askik représente un être qui veut épeler luimême les grandes lignes de son destin: le foyer de vérité est dans l'individu. Et que se passe-t-il dans le roman de V.-L. Beaulieu? Là plane une fatalité, mais qui n'est ni historique ni sociale: la faute de Xavier désigne le noyau de ce déterminisme auquel nul personnage n'échappe, pas même Philippe Couture à Montréal. Appropriation historique, nativité personnelle, faute à purifier: trois voix, trois images d'un même pays.

1 Montréal, Libre Expression, 1987, 494 p.

2 Paris, Albin Michel, 1987, 504 p. Je dois dire en passant que je ne veux pas faire de Ronald Lavallée, qui est de l'Ouest, un écrivain québécois...

3 Montréal, Stanké, 1987, 477 p. 